\title{
Star Formation History in the Fornax Dwarf Galaxy from HST data
}

\author{
G. Marconi, R. Buonanno, M. Castellani, C.E. Corsi \\ Osservatorio Astronomico di Roma \\ R. Zinn \\ Department of Astronomy, Yale University
}

\begin{abstract}
Using observations from the Hubble Space Telescope archive, color-magnitude diagrams have been constructed for globular Cluster 4 in the Fornax dSph galaxy and its surrounding field. These diagrams extend below the main-sequence turnoffs and have yielded measurements of the ages of the populations. In particular, from our analysis Cluster 4 shows $[\mathrm{Fe} / \mathrm{H}] \simeq-2.0$, a value significantly lower than that derived for the Fornax field $([\mathrm{Fe} / \mathrm{H}] \simeq-1.40)$. Putting our results in the frame of the findings of Buonanno et al. (1998) for the Fornax clusters 1, 2, 3 and 5, a global analysis of the properties of Fornax stellar populations has been performed.
\end{abstract}

\section{Introduction}

The dwarf spheroidal (dSph) galaxies of the Local Group provide an opportunity to study star by star the histories of star and star cluster formation in galaxies of the very lowest mass. While once these galaxies were thought to be relatively simple systems composed entirely of very old stars, they are now known to have experienced much more complex histories (see Mateo 1998, for a recent review). The Fornax dSph galaxy is no exception, for it contains very old globular clusters (Buonanno et al. 1998, hereafter BEA98), many stars of intermediate age, and even stars younger than $0.1 \mathrm{Gyr}$ (Stetson 1997). There remain, however, many unanswered questions regarding Fornax, which is one of the most thoroughly studied galaxies of this type. For example, did the star formation in Fornax occur in bursts, as it did in the Carina dSph (Smecker-Hane et al. 1994; Mighell 1997), or was it relatively continuous? Also, what fraction of the field population in Fornax resembles its 5 globular clusters in age and chemical composition?

The most massive dSph galaxies, Fornax and Sagittarius, have their own systems of globular star clusters, which are very interesting objects in their own right. In the case of Fornax, the clusters can be considered to be at essentially the same distance from us, which means that comparisons among them are independent of the uncertain distance scale.

Very recently, BEA98 used the WFPC2 of the Hubble Space Telescope to construct the color-magnitude diagrams (CMDs) of clusters $1,2,3$ and 5 , in order to measure their ages and to explore the connection between $\mathrm{HB}$ mor- 


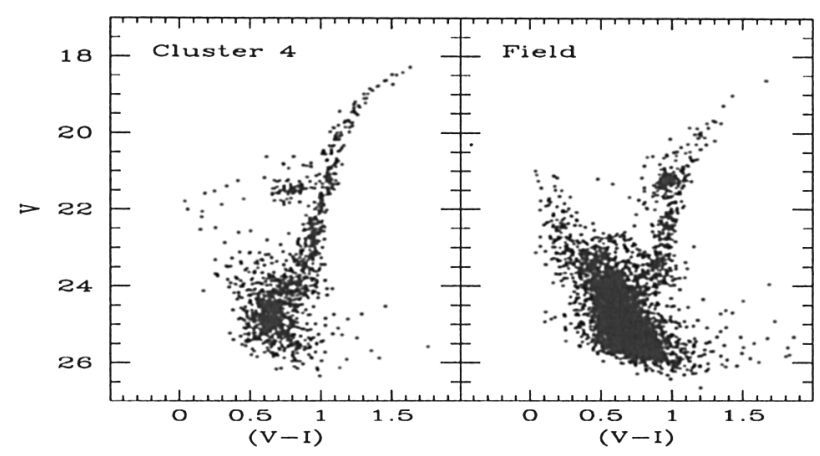

Figure 1. The CMD of Cluster 4 and the field in Fornax.

phology and cluster density. They concluded that the four clusters have the same age to within $1 \mathrm{Gyr}$, and that this age difference is too small to explain the observed differences in HB morphology, unless the HB is more sensitive to age than previously thought. In addition, they noted that a correlation exists between the $H B$ morphologies and the central densities of the clusters.

In this paper we present the results of a study of the CMD of Fornax cluster 4 and its surrounding field. Previous investigations of the CMD of cluster 4 (Buonanno et al. 1985 (BEA85); Beauchamp et al. 1995) have been hampered by the high density of stars in the cluster and in the surrounding field, which is near the center of the galaxy. Because the images from Hubble Space Telescope (HST) have much higher resolution than the previous ones from ground-based telescopes, they have enabled us to do photometry to below the main-sequence turnoff in Cluster 4.

\section{The Field around Cluster 4}

The $V$ vs. $(V-I)$ CMD of the observed stellar field of Fornax is displayed in Fig. 1 (field). As a preliminary, we note that the well-populated sub-giant branch (SGB) indicates the presence of a population older than about $3 \times 10^{9}$ yr because, as discussed by Hardy et al. for the LMC bar, this is the age at which the Hertzsprung gap closes. This evidence, however, does not supply a tight constraint on the age interval of the Fornax field. Moving to a more detailed analysis of data plotted in Fig. 1, we note that the red HB clump shows two important features: (a) the thickness in luminosity, which we estimated to be $\Delta V_{\text {clump }} \simeq 0.5$ and, (b), the large number of stars. These features are due to the "funnelling" effect of the helium-burning red giants older than $1 \mathrm{Gyr}$ and resemble those observed in intermediate age populations in the Magellanic Clouds. Corsi et al. (1994) collected $B, V$ photometry of a sample of 11 clusters in the LMC and estimated several parameters of these clusters. In particular, they measured $V_{c l}$, the average location in $V$ of the red clump, and $V_{T O}$, the location in $V$ of the main sequence turn-off. Adopting now for the LMC $(m-M)_{0}=18.57 \pm 0.05$ (Welch et al. 1987) and $\mathrm{E}(B-V)=0.1$ (Elson \& Fall 1985) we can scale the red clump of the field in Fornax to the LMC distance 
and find that the luminosity of the Fornax clump turns out to be $V_{c l}$ (Fornax to $L M C$ ) $=21.25-21.08+18.89=19.06 \pm 0.25$. This luminosity suggests that the HB clump of the field in Fornax is similar to those of LMC clusters NGC 2173 $\left(V_{\text {clump }}=19.1\right)$, NGC $2162\left(V_{\text {clump }}=19.2\right)$ and NGC $1987\left(V_{\text {clump }}=19.2\right)$. By taking into account data reported by Corsi et al. (1994 see their table 17), we can derive the $V_{T O}$ of these LMC clusters. These results support the evidence that the stars presently forming the red clump are the progeny of a population with about $20.9 \leq V_{T O} \leq 22.1$.

The ages of these clusters can be estimated by adopting the " $s$ " parameter and the calibration provided by Elson \& Fall $(1985,1988)$, i.e.

$$
\log t=0.079 s+6.05 \text {. }
$$

We obtained $t=2.3 \mathrm{Gyr}, 1.4 \mathrm{Gyr}$ and 0.65 Gyr for NGC 2173, NGC 2162 and NGC 1987 respectively, and therefore conclude that the stars presently populating the He-burning clump have an age ranging from 0.5 to 2 Gyr. It is instructive to count the MS stars in the selected interval $20.9 \leq V \leq 22.1, N_{M S}$, and compute the ratio $N_{M S} / N_{\text {clump }}$. This ratio turns out to be $N_{M S} / N_{\text {clump }}=$ $28 / 56=0.50 \pm 0.12$, and can be compared with the same ratio computed from an evolutionary track of the mass corresponding to the age interval $0 \leq t \leq 2$ Gyr. This ratio turns out to be at least an order of magnitude smaller than that found in this paper (S. Cassisi 1998, private communication). In other terms, there are too many stars in the clump! The conclusion is that stars older than 2 Gyr should contribute to the population of RHB clump, as the presence of a well developed SGB independently suggests.

On the other hand, the CMD of Fig. 1 (field) shows that the sub-giant branch (SGB) merges into the MS in a region whose lower limit is near $V \simeq$ 24.35. Assuming that the SGB cannot reach magnitudes brighter than the TO of the oldest population, we conclude that the TO point of the first population of the Fornax field must be located between $V_{T O 1} \simeq 24.35$ and $V_{T O 2} \simeq 24.74$. Using now Eq. 4 of Straniero \& Chieffi (1991), who interpolated their models to derive the visual magnitudes of the turn-off as a function of the age, we transformed into age the limiting magnitudes quoted above, and we found $t_{1}=7.0 \mathrm{Gyr}$ and $t_{2}=10 \mathrm{Gyr}$. In conclusion, the star formation history of the field of Fornax, as derived by the present study, started between 7 and 10 Gyr ago and continued until at least 0.5 Gyr ago.

\section{The Stellar Content of Cluster 4}

The $V$ vs. $V-I$ CMD of Cluster 4 is displayed in Fig. 1 (cluster). The diagram is based on 1343 stars within the distance of 18 arcsec from the cluster center. The overall morphology is similar to that of a Galactic globular cluster, with a well developed RGB extending to $V \simeq 18.28$ and $(V-I) \simeq 1.63$. The subgiant and the TO regions, although clearly delineated, appear somewhat contaminated by the Fornax field. To estimate the metallicity of Cluster 4, we followed the same procedure we used for the Fornax field, and we concluded that the metallicity of cluster 4 is intermediate between M15 and NGC 6397. From linear interpolations we estimated $[\mathrm{Fe} / \mathrm{H}]=-2.01 \pm 0.14$, i.e. a metallicity comparable to those of the other Fornax clusters. The low metallicity we have just found makes a 


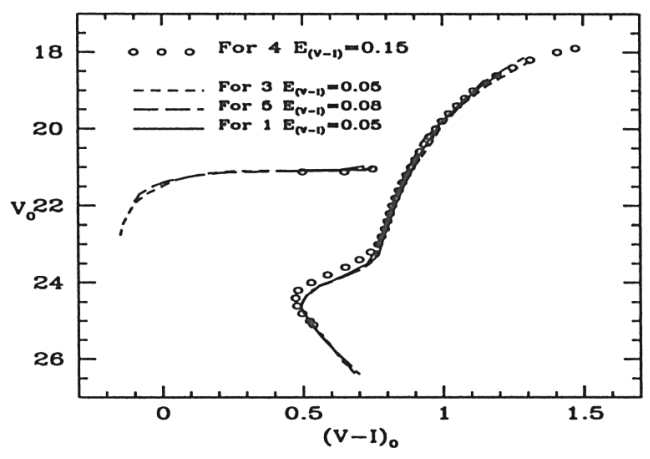

Figure 2. The fiducial lines of Fornax clusters $1,3,4,5$

problem, because it contradicts the much higher metallicity found for Cluster 4 by spectroscopic studies based on its integrated light (Dubath et al. 1992; Zinn \& Persson 1981; Harris \& Canterna 1977; Beauchamp et al. 1995). A further confirmation of the same result is provided by the comparison of the ridge line of Cluster 4 with those of the other GCs in Fornax. We will adopt the technique of Buonanno et al. (1993) which achieves high precision by comparing simultaneously all the relevant branches of the CMDs. This comparison was already performed by BEA98 for clusters 1, 2, 3 and 5 . In Fig. 2 we show the ridge lines of the four Fornax clusters after being shifted by the amounts required by their HB luminosities and reddenings.

Their analysis was based on the estimate of two double differential parameters: $\Delta_{V}$ and $\delta_{(V-I)} . \Delta_{V}$ is defined as $\Delta V_{H B}^{T O}($ ref $)-\Delta V_{H B}^{T O}($ progr $)$, where $\Delta V_{H B}^{T O}$ is the difference in luminosity between the TO point and the HB at the variability strip. The parameter $\Delta_{V}$ is associated with a pair of clusters, the first being the reference cluster and the second the current program cluster. $\delta_{(V-I)}$ is defined as $\Delta(V-I)_{T O}^{R G B}($ ref $)-\Delta(V-I)_{T O}^{R G B}($ progr $)$, where $\Delta(V-I)_{T O}^{R G B}($ ref $)$ is the color difference between the TO and the base of the RGB and is the equivalent in the $V,(V-I)$ plane of the $\delta(B-V)$ defined by Vandenberg et al. $(1990)$ in the $V,(B-V)$ plane. Since the Fornax clusters have nearly the same metallicity, the analysis was particularly straightforward.

The mean age difference is then

$$
\begin{aligned}
& \Delta \mathrm{t}\left(\text { cl.4 }- \text { cl.1,3,5) }=-2.97 \pm 0.54\left(\text { from } \Delta_{V}\right)\right. \\
& \Delta \mathrm{t}\left(\text { cl.4 }- \text { cl.1,3,5) }=-2.83 \pm 0.46\left(\text { from } \delta_{(V-I)}\right)\right.
\end{aligned}
$$

where the associated error is 3 times the standard error, $\sigma$. From the mean of the two determinations we conclude that Cluster 4 appears to be about $2.9 \mathrm{Gyr}$ younger than the other Fornax clusters.

\section{References}

Beauchamp, D., Hardy, E., Suntzeff, N.B., Zinn, R. 1995, AJ, 109, 1628

Buonanno, R., Corsi, C.E., Fusi Pecci, F., Hardy, E., Zinn, R. 1985, A\&A, 152, 65 (BEA85) 
Buonanno, R., Corsi, C.E., Fusi Pecci, F., Richer, H.B., Fahlman, G.G. 1993, AJ, 105, 184

Buonanno, R., Corsi, C.E., Zinn, R., Fusi Pecci, F., Hardy, E., Suntzeff, N.B. 1998, ApJ, 501, 33L (BEA98)

Corsi, C.E., Buonanno, R., Fusi Pecci, F., Ferraro, F.R., Testa, V., Greggio, L. 1994, MNRAS, 271, 385

Dubath, P., Meylan, G., Mayor, M. 1992, ApJ, 400, 510

Elson, R.A.W., Fall, S.M. 1985, ApJ, 299, 211

Elson, R.A.W., Fall, S.M. 1988, AJ, 96, 1383

Hardy, E., Durand, D. 1984, ApJ, 279, 567

Harris, H.C., Canterna, R. 1977, AJ, 82, 798

Mateo, M. 1998, ARAA, 36, 435

Mighell, K.J. 1997, AJ, 114, 1458

Smecker-Hane, T.A., Stetson, P.B., Hesser, J.E. 1994, AJ, 108, 507

Stetson, P.B. 1997, Baltic Astronomy, 6, 3

Straniero, O., Chieffi, A. 1991, ApJS, 76, 525

VandenBerg, D.A., Bolte, M., Stetson, P.B. 1990, AJ, 100, 445

Welch, D.L., McLaren, R.A., Madore, B.F., McAlary, C.W. 1987, ApJ, 321, 162

Zinn, R., Persson, S.E. 1981, ApJ, 247, 849 